\title{
BASIC SCIENCE ARTICLE Differential cell proliferation and cell death during the urethral groove formation in guinea pig model
}

\author{
Shanshan Wang ${ }^{1}$ and Zhengui Zheng ${ }^{1}$
}

BACKGROUND: Urethral groove (UG) formation is an important step in penile formation. Because commonly used animal models do not have UG, the mechanisms of UG formation have never been discovered. We aim to discover the cellular mechanism of the UG formation using guinea pig model.

METHODS: Histology was used to study the ontogeny of UG. BrdU immunofluorescence was used to label proliferating cells, cell death was determined using LysoTracker Red and TUNEL staining, and stereology was used for quantification. To reveal Shh mRNA expression patterns, in situ hybridization was performed in guinea pig genital tubercles (GTs) and Shh ${ }^{\text {GFPcre }}$-LacZ-reporter mice were used for comparison.

RESULTS: Cell proliferation in the outer layers and programmed cell death in the inner layers of urethral epithelium played key roles during urethral canal movement from dorsal to ventral aspect and final opening to form UG. Shh mRNA expression domain shifted out to the ventral surface of GT from proximal throughout to distal in guinea pigs, but was excluded from the ventral surface epithelium in midshaft and distal of mouse GT.

CONCLUSION: Differential cell proliferation and cell death in developing urethral epithelium lead to UG formation and Shh expression in ventral surface epithelium of GT may play an important role.

Pediatric Research (2019) 86:452-459; https://doi.org/10.1038/s41390-018-0236-0

\section{INTRODUCTION}

Hypospadias is the most common congenital anomaly of the urogenital system, which affects approximately 1:200 male live births. ${ }^{1,2}$ The incidence of hypospadias keeps increasing in the past decades. ${ }^{3,4}$ The etiology of hypospadias is largely unknown and the leading hypothesis is that both genetic susceptibility and environmental exposure cause this anomaly. ${ }^{5,6}$ Hypospadias is an ectopic ventral opening of the urethra and the malformation is formed during external genital sex differentiation. In order to discover which mechanisms are being perturbed in children with hypospadias and reveal whether the cause of the defect is genetic, environmental, or a combination of both, we first need to understand the tubular urethral development in normal males.

The most important step in sex differentiation of external genitalia is the tubular urethra formation in males. In humans, the male genital tubercle (GT) development involves an initial opening of the urethral plate to form urethral groove (UG) from proximal to distal, and subsequent fusion of the proximal region to close the urethral and penile epithelia and extend the closed urethra distally (distal opening proximal closing zipper). ${ }^{7}$ The females show the similar urethral plate opening to form UG, but lack of the following urethral closure seen in males (the single opening zipper). ${ }^{8}$ Both males and females form UGs before external genital sex differentiation.

In humans, canalized urethral plate has been observed before the UG formed, but very little is known about the mechanism underlying canalization of the urethral plate and how it gives rise to UG. It is interesting that no CASP3-positive cells were detected at the time of UG formation. ${ }^{7}$ If apoptosis is not involved in this process, what other cellular mechanisms play the role? Is another type of cell death involved? Cell proliferation in the urethral plate, urethral canal, as well as the UG has been reported ${ }^{7,8}$, but little has been known about how does cell proliferation lead to the urethral canal and the UG formation. In this study, we try to answer these fundamental questions by testing the hypothesis that differential proliferation and death of urethral epithelial cells among different regions of the urethral plate lead to UG formation using the guinea pig model.

\section{MATERIALS AND METHODS}

Animal breeding and embryo collection

Fifty to sixty days old male and female Hartley guinea pigs were purchased from Elm Hill Labs, and CD1 mice were purchased from Charles River. All experiments were conducted in accordance with the "Guide for the Care and Use of Laboratory Animals", and the experimental protocols were approved by the Institutional Animal Care and Use Committee of Southern Illinois University Carbondale. Timed mating of guinea pigs using the procedure previously described $^{9}$, we crossed males and females once the vaginal closure membrane opened, and the time that a vaginal plug or vaginal sperm smear detected was designated as embryonic day (E)0 (As guinea pig is not strictly nocturnal, we observed most of them plugged during daytime, here we use E0, not E0.5). For mice, females were inspected for vaginal plugs, and the morning they were found was determined as E0.5. A minimum of three litters embryos were collected at each stage and GTs were dissected and photographed under a stereoscope (Leica M80). The Shh ${ }^{\text {GFPcre }}$ and Rosa26 reporter (R26R) mice used in this study were purchased from Jax laboratory and have been described previously. ${ }^{10,11}$ To

${ }^{1}$ Department of Physiology, School of Medicine, Southern Illinois University Carbondale, Carbondale, IL 62901, USA

Correspondence: Zhengui Zheng (zzheng57@siumed.edu)

Received: 26 March 2018 Accepted: 28 October 2018

Published online: 22 November 2018 
label $S h h^{\text {GFPcre }}$-expressing cells, we crossed heterozygous $\left(S h h^{\text {GFPcre+/-) }}\right.$ males to females carrying the R26R reporter allele. Pregnant dams were sacrificed at specific times to collect staged embryos with $S h h^{G F P c r e} ; R 26 R$ genotypes, and embryos were then processed for X-gal staining and histological analysis.

Histology and X-gal staining

Guinea pig specimens were harvested and washed in PBS, and then fixed overnight in 4\% PFA at room temperature. For $\mathrm{Shh}^{\text {GFPCre; }}$ $R 26 R$ reporter mice to detect $\beta$-galactosidase activity, mouse embryos were harvested in PBS and fixed overnight in $0.2 \%$ PFA at $4{ }^{\circ} \mathrm{C}$, then washed three times in LacZ rinse buffer, and stained with X-gal overnight rocking at room temperature. ${ }^{12}$ Embryos were then rinsed, post fixed, and stored in $4 \%$ PFA at $4{ }^{\circ} \mathrm{C}$ afterward. The post-fixed samples were processed through several changes in graded ethanol, and Histo-Clear II (Electron Microscopy Sciences), and then embedded in paraffin wax. Sections were cut at 6-8 $\mu \mathrm{m}$ and stained with hematoxylin and eosin for histology, or only counterstained with eosin for lacZ-stained samples.

\section{In situ hybridization}

Three embryos of each stage were selected for in situ hybridization analysis. In situ hybridization was performed as described ${ }^{13}$ with the following modifications: embryos were bleached in $6 \%$ hydrogen peroxide in methanol for $30 \mathrm{~min}$ after rehydration, and fixed in $4 \%$ PFA plus $0.2 \%$ glutaraldehyde for $30 \mathrm{~min}$ immediately after treatment with proteinase $\mathrm{K}(5 \mu \mathrm{g} / \mathrm{ml}, 5 \mathrm{~min}$ for E22-E25, 7 min for E27 embryos). For color reaction, $10 \%$ dimethyl formamide (DMF) was added to NBT/BCIP solution. To make the Shh RNA probe, Shh CDNA was PCR-amplified from E20-22 guinea pig CDNA using the following primers: forward: $5^{\prime}$-TGC TGC TGG CGA GAT GT-3', reverse: 5'-GAA GGT GAG GAA GTC GCT GTA G-3', and the product was cloned to a TA vector using InsTAclone PCR cloning kit (Thermo Scientific, K1214). The antisense probe was made using T7 RNA polymerase-mediated transcription reaction and purified using SigmaSpin Sequencing Reaction Clean-Up columns (Sigma-Aldrich, S5059). After coloring, and photography under the stereoscope (Leica), the fixed GTs were embedded in paraffin wax. Ten micrometer sections were cut and counterstained with eosin, and then photographed.

\section{BrdU incorporation and LysoTracker assay}

The cell proliferation and programmed cell death assays in mice were performed as described previously. ${ }^{14}$ To detect cell proliferation in guinea pigs, 5-bromo-2'-deoxyuridine (BrdU, 50 $\mathrm{mg} / \mathrm{kg}$ ) was injected intraperitoneally and embryos were collected $4 \mathrm{~h}$ later for immunohistochemistry. LysoTracker Red DND-99 (Life Technologies, L7528) staining was used to detect programmed cell death in guinea pig GTs following the published method. ${ }^{15}$ In brief, dissected GTs were stained with LysoTracker Red before fixation. After being washed twice in pre-warmed PBS $\left(37^{\circ} \mathrm{C}\right)$, the samples were incubated in $5 \mu \mathrm{M}$ LysoTracker Red staining solution at $37^{\circ} \mathrm{C}$ for $30 \mathrm{~min}$, followed by washing with $37^{\circ} \mathrm{C}$ PBS 3-4 times and fixed in $4 \%$ PFA at $4{ }^{\circ} \mathrm{C}$ overnight, and then dehydrated through a methanol series $(50 \%, 75 \%, 95 \%$, and $100 \%, 10-20$ min each step) to eliminate background staining. ${ }^{15}$ Then the samples were embedded in paraffin, and $10-\mu \mathrm{m}$ sections were cut, stained with DAPI, and photographed on a fluorescence microscope (Leica). A minimum of three litters at each stage, and at least two embryos from each litter were selected for BrdU or LysoTracker analysis.

BrdU immunofluorescence and TUNEL staining

Immunofluorescence of BrdU was performed according to standard immunofluorescence protocol. In brief, after $2 \mathrm{~N} \mathrm{HCl}$ treatment for $30 \mathrm{~min}$, selected sections of tissue were incubated in mouse anti-BrdU (1:10, G3G4, Developmental Studies Hybridoma Bank, $A B$ 2314035) overnight at $4{ }^{\circ} \mathrm{C}$ and then in the minimally a
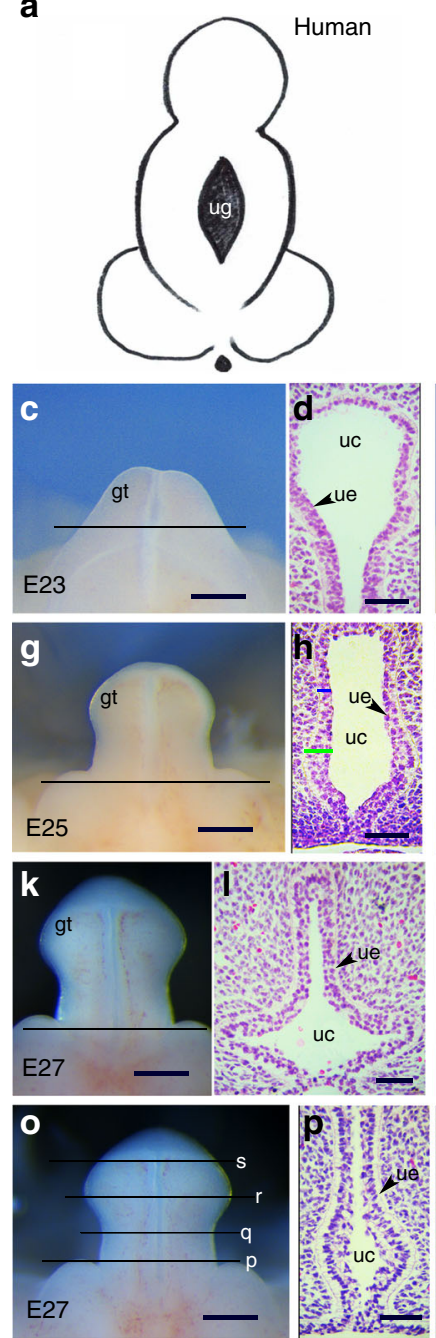

b

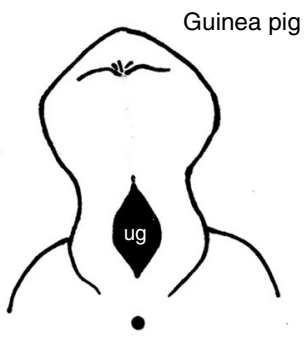

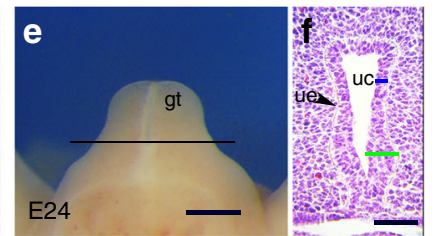
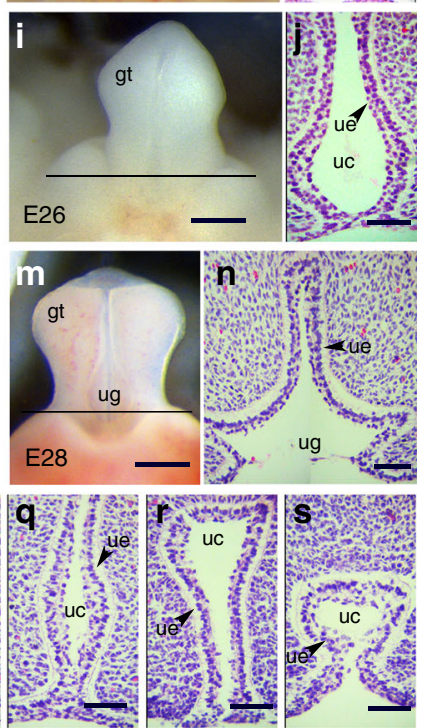

Fig. 1 Ontogeny of guinea pig urethral groove (UG). Schematic drawings of the ventral views of embryonic 9.5 weeks human (a) and embryonic 28 days guinea pig (b) genital tubercles (GTs) with distal at the top, showing similar developing UG. Images in $\mathbf{c}, \mathbf{e}, \mathbf{g}, \mathbf{i}$, $\mathbf{k}, \mathbf{m}$, and $\mathbf{o}$ are ventral views of guinea pig GTs with distal at the top. The stage is indicated at down-left. All sections are transverse through GT with dorsal at the top. Panels $\mathbf{d}, \mathbf{f}, \mathbf{h}, \mathbf{j}, \mathbf{I}$, and $\mathbf{n}$ are showing the proximal sections through positions indicated by the dark lines on images of $\mathbf{c}, \mathbf{e}, \mathbf{g}, \mathbf{i}, \mathbf{k}$, and $\mathbf{m}$, respectively. Shorter blue and longer green lines marked on urethral epithelium of $\mathbf{f}$ and $\mathbf{h}$ showing the epithelial thickness changes. Dark lines ( $p, q, r$, and $s$ ) on image (o) indicate the planes of sections shown in $\mathbf{p}, \mathbf{q}, \mathbf{r}$, and $\mathbf{s}$, respectively. gt genital tubercle, uc urethral canal, ue urethral epithelium, ug urethral groove. Scale bars in GT images (c, e, g, i, $\mathbf{k}$, $\mathbf{m}, \mathbf{o})$ and histological sections (d, f, h, j, $\mathbf{l}, \mathbf{n}, \mathbf{p}, \mathbf{q}, \mathbf{r}$, and $\mathbf{s}$ ) are 500 and $50 \mu \mathrm{m}$, respectively.

cross-reactive secondary Alexa Fluor 488 (or 546) goat anti-mouse IgG (1:500, Life Technologies, A11001; A21123) antibody at $4{ }^{\circ} \mathrm{C}$ overnight. The tissues were washed repeatedly between steps with PBS, then stained with DAPI, and mounted. For double labeling of BrdU and TUNEL [terminal deoxynucleotidyl transferase (TdT) dUTP nick end labeling], the recombinant TdT-mediated incorporation of fluorescein-12-dUTP was performed using DeadEnd $^{\mathrm{TM}}$ Fluorometric TUNEL system (Promega, G3250) according to the manufacturer's instructions first, followed by BrdU immunostaining on the same tissue section. Fluorescence microscopy was performed on a confocal microscope (Leica DM5500Q). A 
454

minimum of three litters at each stage, and slides obtained from at least two embryos from each litter were selected for BrdU immunostaining, and E26 embryos were used for BrdU and TUNEL double labeling.
Stereology and quantification

Multiple serial sections were performed for cell proliferation and cell death assays, and stereology was used to determine the total cell proliferation and cell death in dorsal and ventral regions.
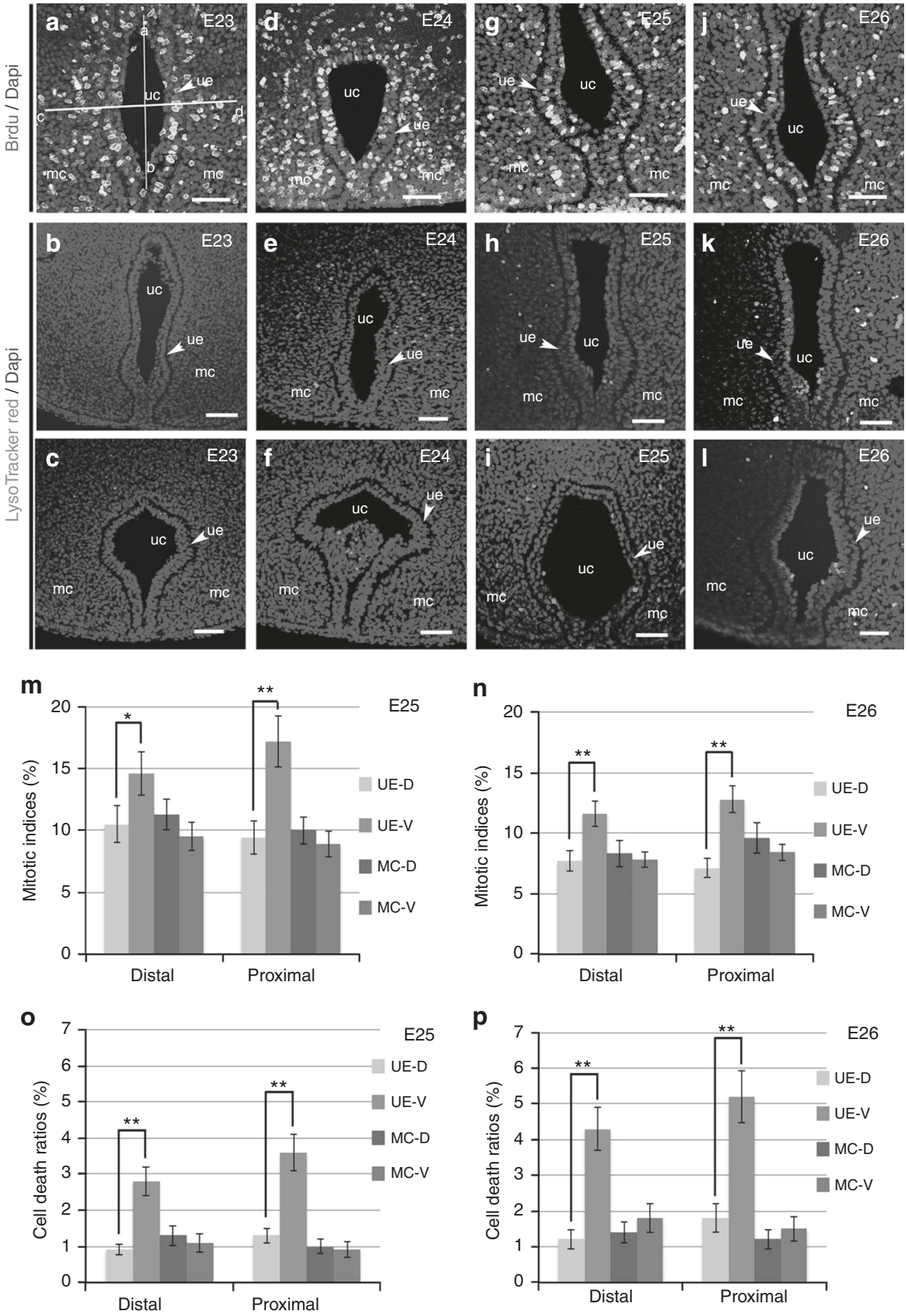

Fig. 2 Dorsal-ventral variations of BrdU and LysoTracker positive cells in urethral epithelia and the adjacent mesenchyme during GT development. Images (a-I) are transverse sections ( $\mathbf{a}, \mathbf{d}, \mathbf{g}, \mathbf{j}, \mathbf{c}, \mathbf{f}, \mathbf{i}$, and I: proximal; $\mathbf{b}, \mathbf{e}, \mathbf{h}$, and $\mathbf{k}$ : more distal) of GT of different stages (labeled up-right) with dorsal on the top, showing signals of BrdU (green in a, d, g, and j), LysoTracker (red in b, c, e, f, h, i, $\mathbf{k}$, and I), and DAPI (blue in $\mathbf{a}-I) . \mathbf{m}, \mathbf{n}$ The comparison of BrdU-positive cells (mitotic indices) between dorsal and ventral of urethral epithelium (UE-D vs UE-V) and adjacent mesenchyme (MC-D vs MC-V) in distal and proximal regions of E25 (m) and E26 (n) guinea pig GTs, the dorsal or ventral was determined using a line cross through the middle point of dorsal-ventral diameter of urethral lumen (such as line segment cd in a, ab is the dorsal-ventral diameter of urethral lumen). o, $\mathbf{p}$ The comparison of LysoTracker positive cells (cell death ratios: dying cells/total cells) between dorsal and ventral of urethral epithelium (UE-D vs UE-V) and adjacent mesenchyme (MC-D vs MC-V) in distal and proximal regions of E25 (o) and E26 (p) guinea pig GTs, and the dorsal or ventral was determined in the same way as $\mathbf{m}$ and $\mathbf{n}$. The data in $\mathbf{m}-\mathbf{p}$ are mean $(n=6) \pm$ standard error (SE), ${ }^{*} p<0.05,{ }^{* *} p<0.01$. mc mesenchyme, uc and ue are the same as Fig. $1 ; \mathrm{D}$ dorsal, $\mathrm{V}$ ventral. Scale bars in a-I are $50 \mu \mathrm{m}$. 
Mitotic indices and cell death ratios were compared between dorsal and ventral regions and among different stages. Total (DAPI-labeled), BrdU or LysoTracker labeled cells were estimated stereologically using the optical fractionator method (under a confocal microscope) by counting target cells on every 12th $3-\mu \mathrm{m}$ transverse optical section (about 12-24 sections through the entire proximal-distal axis of GT at different stages (E24-E28)), following published methods for counting cells through irregularly shaped structures, such as brain regions or kidneys. ${ }^{16-18}$ Briefly, cells of interest were counted on image stacks acquired using a $10 x$ objective, with Image-J software. Cells that fell within the $45 \times$ $45-\mu \mathrm{m}$ counting frames were used in the analyses. To estimate total target cells in the mesenchyme, the area of the mesenchyme on each section was traced and quantified using Image-J and the total volume of the structure was estimated using Cavalieri's principle. ${ }^{16}$ Densities were determined by dividing the number of cells in the region of interest by the area in which the cells were counted (i.e., $45 \times 45 \mu \mathrm{m}$ ). Proportions of cells were calculated by counting total cells (DAPI-labeled) and BrdU antibody or LysoTracker red labeled cells in five separate counting frames per portion of section (dorsal or ventral) for mesenchyme, labeled urethral epithelial cells were counted on the entire section to avoid subsampling errors. ${ }^{18}$

Statistical analysis

The variances between dorsal and ventral regions, or inner and outer layers and among different stages were evaluated using ANOVA with a significance level set at 0.05 .

\section{RESULTS}

The movement of the urethral canal from dorsal to ventral along the urethral plate led to UG formation

In guinea pig external genital development, both males and females form UG, similar to humans (Fig. 1a, b). External genital development begins with the emergence of two genital swellings in mice and guinea pigs. ${ }^{9,19}$ When the two swellings fuse to form a single structure: GT, the gut epithelium and urethral epithelium form a urethral plate. In guinea pigs, the urethral plate has an enlarged canal located at its dorsal end in proximal GT at the stage of E22 ${ }^{9}$ and E23 (Fig. 1C, d). On E24, the urethral canal is still in the dorsal aspect, but the number of cell layers of the urethral plate epithelium in the ventral aspect is increased, so that the urethral epithelium in the ventral aspect is more than twice as thick as in dorsal (Fig. 1e, f). The enlarged urethral canal in the proximal region moves to ventral at $\mathrm{E} 25$, and the thickened urethral plate epithelium is still obvious at this stage (Fig. $1 \mathrm{~g}, \mathrm{~h}$ ). In proximal GT, the urethral canal reaches the ventral end of the urethral plate on E26 (Fig. 1i, j). It keeps enlarging and moving ventrally, only a few layers of urethral seams remain covered on the ventral margin in the proximal region at E27, but now the canal's opening is imminent (Fig. $1 \mathrm{k}, \mathrm{I}$ ). The ventral side of the urethral plate starts to open and forms UG in the proximal region at E28 in both males and females (Fig. $1 \mathrm{~m}, \mathrm{n}$ ). In both humans and guinea pigs, the UG opening starts from proximal and then distends distally.,8,9 Using E27 guinea pig GT as an example, we then observed the serial transverse sections, and it was revealed that the enlarged urethral canal was located from ventral of the urethral plate in proximal gradually to the dorsal in distal (Fig. 10-s).

Differential cell proliferation and cell death in urethral epithelium played important roles during urethral canal movement from dorsal to ventral

In order to understand how the urethral canal moved from dorsal to ventral along the urethral plate, we examined cell proliferation and programmed cell death in developing guinea pig GTs. When the urethral canal was located at proximal dorsal urethral plate on E23 and E24, lots of BrdU-labeled proliferating cells were found in
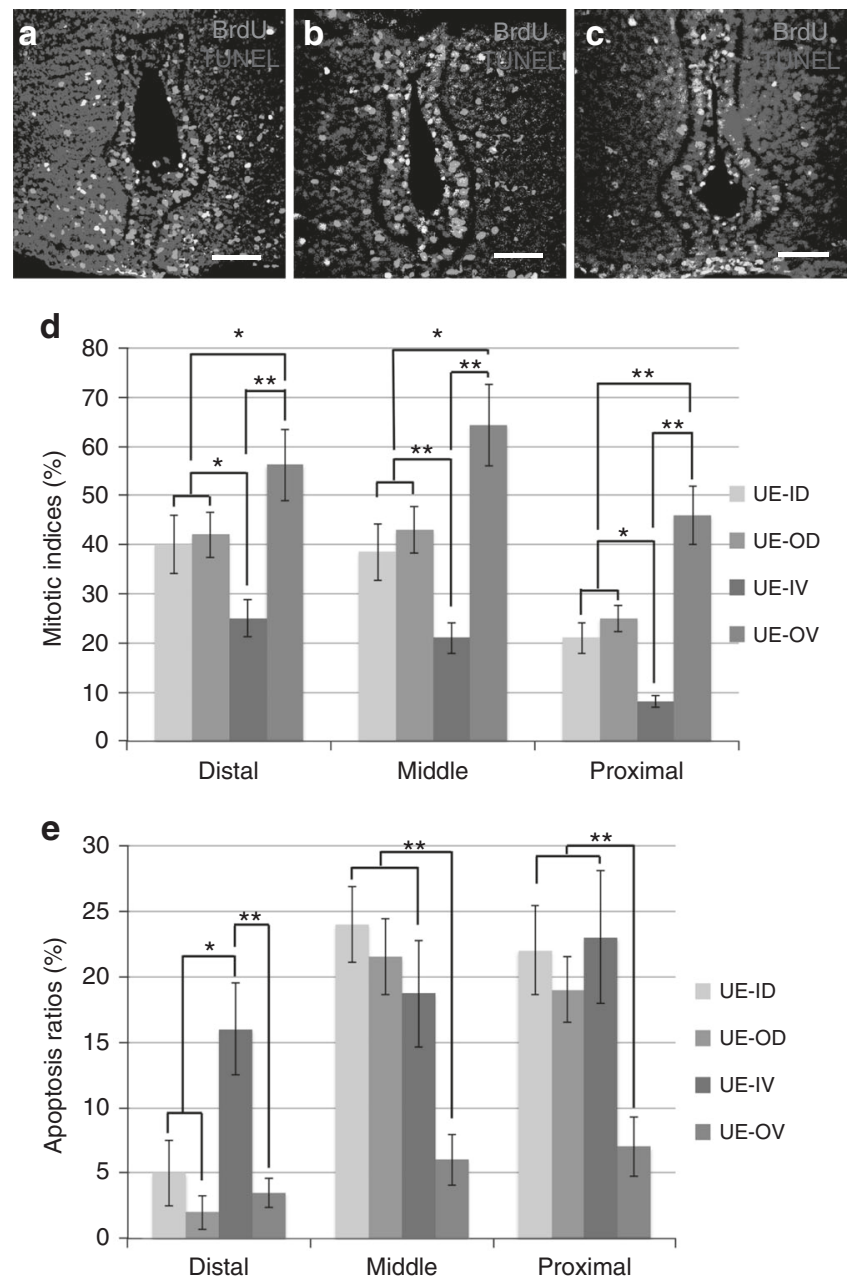

Fig. 3 The variations of apoptosis and cell proliferation in different layers (inner vs outer) of urethral epithelium in E26 guinea pig GT. Images (a-c) are transverse sections of distal (a), middle (b), and proximal (c) regions of E26 GT with dorsal on the top, showing TUNEL (green), BrdU (red), and Dapi (blue). Panels d, e show the comparison of cell proliferation (d) and apoptosis (e) between inner and outer layers of urethral epithelium in dorsal (UE-ID vs UE-OD) and ventral (UE-IV vs UE-OV) regions of E26 GT. We selected one basal layer (total epithelium $\leq 3$ layers) or the outmost two layers (total epithelium $>3$ layers) of epithelium as outer layers, the inner layers were defined on the contrary way, and the dorsal or ventral was determined in the same way as (Fig. $2 \mathrm{~m}$ ). The data in $\mathbf{d}$ and $\mathbf{e}$ are mean $(n=4) \pm$ standard error $(\mathrm{SE}),{ }^{*} p \leq 0.05,{ }^{* *} p \leq 0.01$. ID inner, dorsal, OD outer, dorsal, IV inner, ventral, OV outer, ventral, UE is the same as Fig. 1 or Fig. 2. Scale bars in a-c are $50 \mu \mathrm{m}$.

different layers of urethral epithelium around the canal, and no significant difference between dorsal and ventral in both urethral epithelium and the adjacent mesenchyme ( $p \geq 0.086$, Fig. $2 a$, d, and Fig S1A, B). At these stages, very few LysoTracker ${ }^{\circledast}$ Red positive cells were detected in urethral epithelium in either dorsal or ventral region ( $p \geq 0.275$, Fig. $2 \mathrm{~b}, \mathrm{c}, \mathrm{e}, \mathrm{f}$ and Fig. S1C). There was some cell death detected in mesenchymal cells of E24 (only a few in E23) tubercle, but no significant difference was found between dorsal and ventral sides ( $p \geq 0.102$, Fig. 2b, c, e, f and Fig. S1D). When the urethral canal started moving ventrally, the cell proliferation in dorsal portion of urethral epithelium decreased, more BrdU-positive cells were found in the ventral part of urethral epithelium at E25 and E26, majority of them were located in the thickened region where increased layers formed, and especially in the outer layers ( $p \leq 0.014$ for E25 and $p \leq 0.006$ for E26, Fig. $2 \mathrm{~g}, \mathrm{j}$, $m, n)$. No significant dorsal-ventral difference was found in 

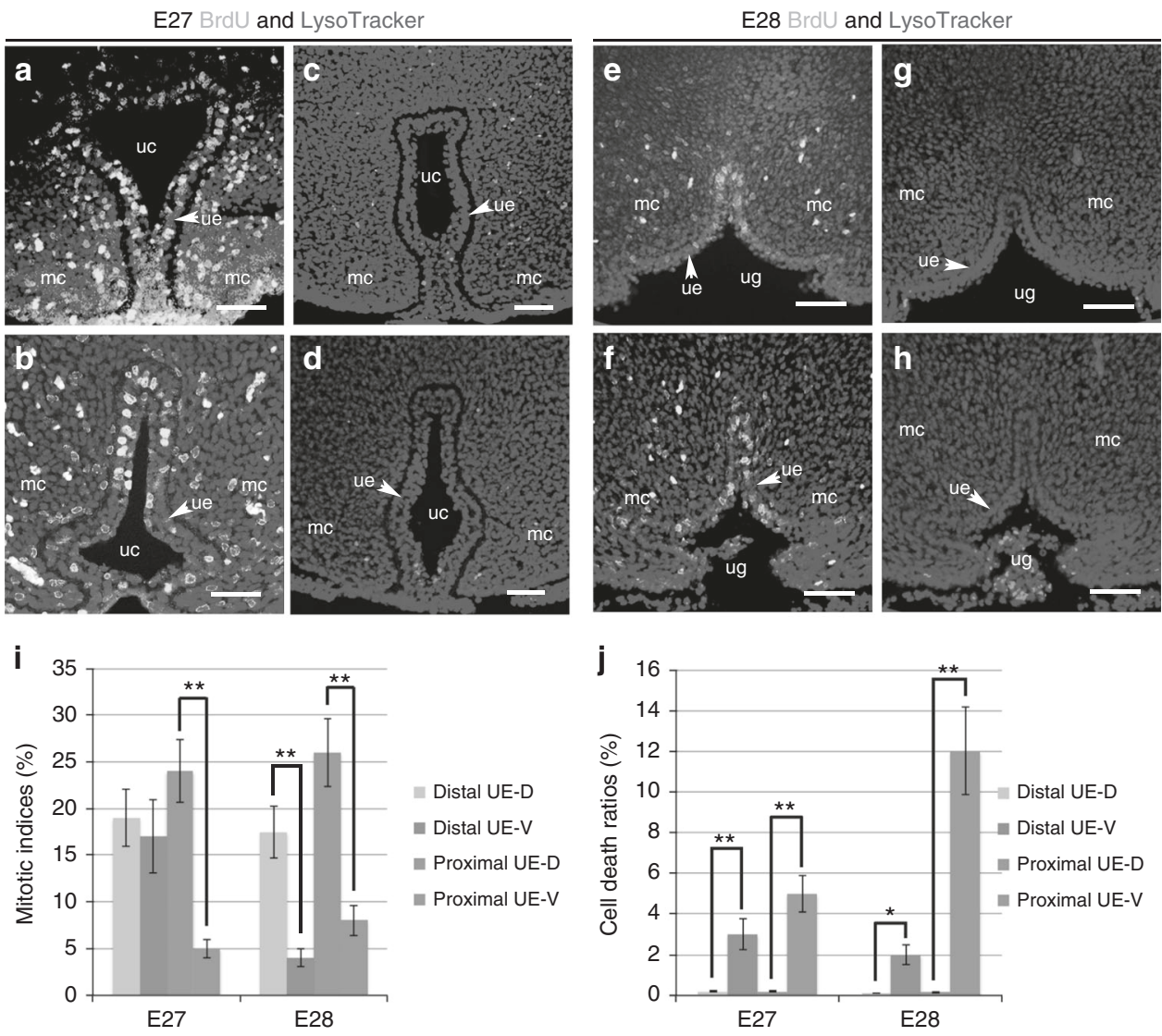

Fig. 4 Urethral epithelial cell proliferation and cell death during UG formation in guinea pigs. Images (a-h) are transverse sections of proximal $(\mathbf{b}, \mathbf{d}, \mathbf{f}$, and $\mathbf{h})$ and more distal (a, c, e, and $\mathbf{g})$ GT of E27 (a-d) and E28 (e-h) with dorsal on the top, showing signals of BrdU (green in $\mathbf{a}, \mathbf{b}, \mathbf{e}$, and f), LysoTracker (red in $\mathbf{c}, \mathbf{d}, \mathbf{g}$, and $\mathbf{h}$ ), and DAPI (blue in $\mathbf{a}-\mathbf{h})$. Panels $\mathbf{i}$, $\mathbf{j}$ show the comparison of cell proliferation (i) and death (j) between dorsal and ventral of urethral epithelium in distal and proximal regions (UE-D vs UE-V) of developing GT, and the dorsal or ventral was determined in the same way as in Fig. $2 \mathrm{~m}$. The data in $\mathbf{i}$ and $\mathbf{j}$ are mean $(n=6) \pm$ standard error (SE), ${ }^{*} p<0.05,{ }^{* *} p<0.01$. mc, uc, ue, ug, D, and $V$ are the same as in Figs. 1 and 2. Scale bars in $\mathbf{a}-\mathbf{h}$ are $50 \mu \mathrm{m}$.

mesenchymal cell proliferation in either E25 $(p \leq 0.256)$ or E26 ( $p \leq$ 0.138 ) GT (Fig. 2g, j, $m$, and n). Interestingly, we also found more LysoTracker Red positive cells in the inner layers of the ventral urethral epithelium in sections through both proximal and distal regions of E25 $(p \leq 0.006)$ and E26 $(p<0.001)$ (Fig. 2h, i, k, l, o, p) GTs. Similar to cell proliferation, no significant dorsal-ventral difference was found in mesenchymal cell death in either E25 $(p \leq$ $0.255)$ or E26 ( $p \leq 0.106) \mathrm{GT}$ (Fig. $2 \mathrm{~h}, \mathrm{i}, \mathrm{k}, \mathrm{l}, \mathrm{o}, \mathrm{p})$. The results suggest that differential cell proliferation and cell death between dorsal and ventral of urethral epithelium may contribute to the urethral canal movement from dorsal to ventral along the urethral plate.

LysoTracker labels both apoptosis and autophagy. ${ }^{15,20}$ To determine whether apoptosis exists in UG formation process and to show cell proliferation and apoptosis on the same section, we performed BrdU and TUNEL double staining on transverse sections of E26 guinea pig GT. Our data showed obvious inner layer apoptosis and outer layer proliferation of urethral epithelium in the ventral aspect of the urethral canal in proximal, middle, and distal of E26 GT (Fig. 3a-C). We compared the mitotic indices and apoptosis ratios among four different regions (ID, inner layer, dorsal; OD, outer layer, dorsal; IV, inner layer, ventral; and OV, outer layer, ventral) of urethral epithelium, and the data suggested that the most and least cell proliferation were found in OV and IV, respectively, which showed significant differences from other groups ( $p \leq 0.034$, Fig. 3a-d). In addition, most of apoptotic cells in the distal sections were detected in IV region, significantly higher than in other regions ( $p \leq 0.012$, Fig. $3 a, e)$. In the middle and proximal sections, the least of apoptotic cells were detected in OV, significantly less than in other regions $(p \leq 0.004)$, but both ID and OD showed apoptotic cells similar to those in IV ( $p \geq 0.251$, Fig. $3 b$, $c$, and e), suggesting that apoptosis in dorsal region may contribute to the observed whole urethra movement from dorsal to ventral during development.

Programmed cell death in urethral epithelium was observed in the final UG opening process

To understand the UG opening process, we also performed cell proliferation and programmed cell death assays on E27 and E28 guinea pig GTs. In the distal region of E27 GT, the urethral canal was located in dorsal aspect and many BrdU-positive cells were found in different layers of urethral epithelium (Fig. 4a), and no significant dorsal-ventral differences of mitotic indices were found ( $p=0.256$, Fig. $4 \mathrm{i})$. More BrdU-positive cells were seen in the dorsal aspect of urethral epithelium than in ventral in proximal region ( $p=0.004$, Fig. $4 \mathrm{~b}, \mathrm{i}$ ). The LysoTracker positive cells were found mainly in the inner layers of ventral aspect of urethral epithelium in both distal and proximal regions $(p<0.001$, Fig. 4c, d, j). In E28 GT, many more BrdU-positive cells were detected in the dorsal aspect than in ventral $(p<0.001$, Fig. $4 \mathrm{e}, \mathrm{f}, \mathrm{i})$. In a more distal region, LysoTracker Red positive cells were exclusively found on both sides of the epithelial junction of a newly exposed urethral epithelium and surface epithelium on the ventral side of GT where the UG opened ( $p=0.017$, Fig. $4 \mathrm{~g}, \mathrm{j}$ ). Many more LysoTracker positive cells were observed on the ventral side of E28 GT in the proximal region ( $p<0.0001$, Fig. $4 \mathrm{~h}, \mathrm{j})$. 

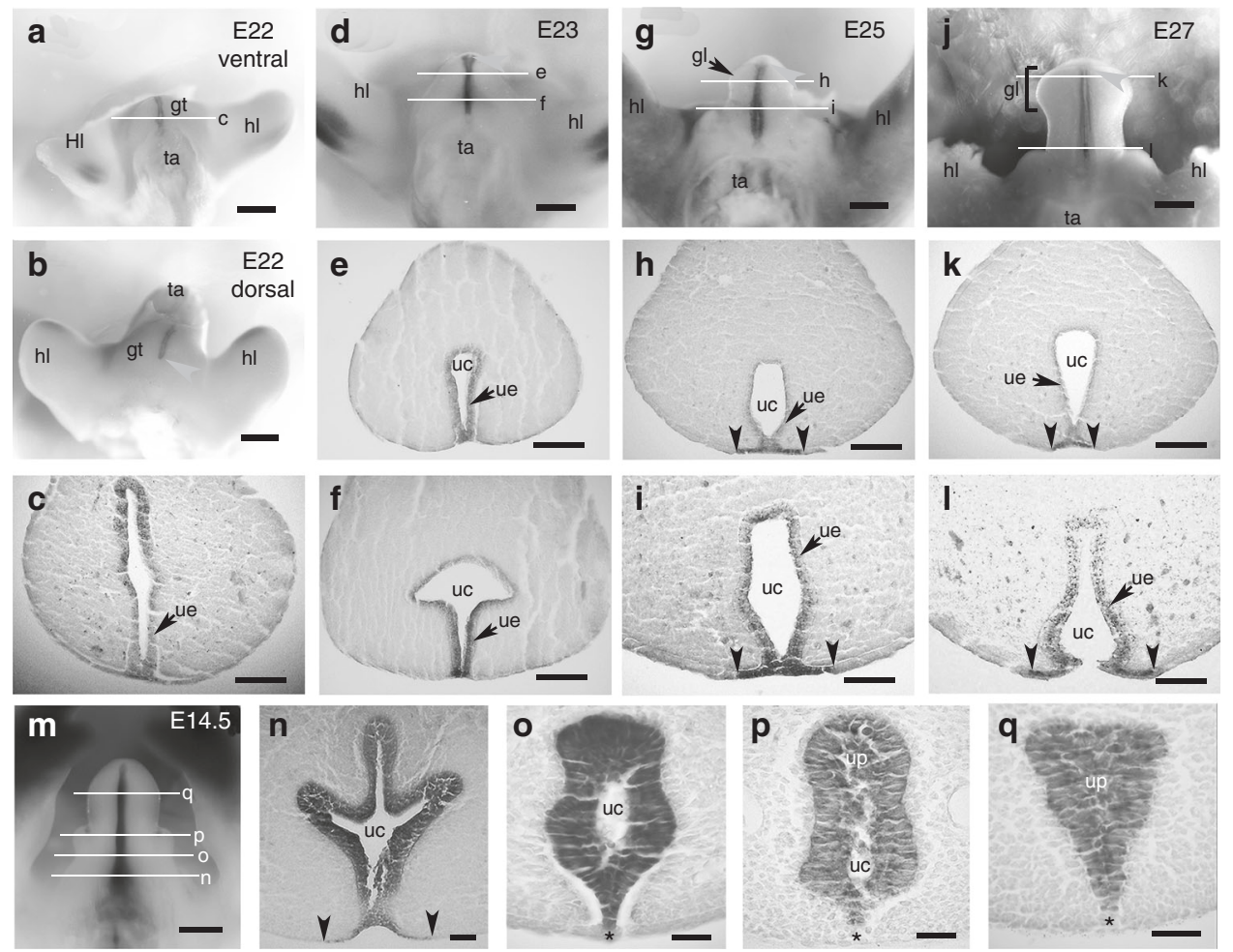

Fig. 5 Shh mRNA expression in developing guinea pig and mouse GTs. Shh in situ hybridization was performed on guinea pig GTs of different stages, and Shh expression domain was stained blue (a-I). X-gal stained E14.5 Shh ${ }^{G F P C r e}$ LacZ mouse GT was used to show mouse Shh expression domain (blue, $\mathbf{m}-\mathbf{q})$ for comparison. Images (a, $\mathbf{d}, \mathbf{g}$, and $\mathbf{j}$ ) are ventral views of guinea pig GTs, b is dorsal view of guinea pig GT. Panel $\mathbf{m}$ is ventral view of mouse GT. Gestation stage is labeled at up-right. Green arrowheads in $\mathbf{b}$, $\mathbf{d}, \mathbf{g}$, and $\mathbf{j}$ point to the dorsal (b) or distal end (d, $\mathbf{g}$, and $\mathbf{j}$ ) of Shh expression domain. Dorsal is on the top in all sections. a-c Shh expression domain is in urethral plate, extending from ventral (a) to dorsal (b) of GT at E22, and is displaced to the ventral side of GT by E23 (d). Panel c is transverse section of GT in a through the level indicated by the white line (c). Shh expression domain remains in the whole urethra plate and extends out to the ventral surface of GTs at E25 and E27, this extension of Shh domain exists from proximal throughout to distal of guinea pig GTs (g-I). (e, h, and $\mathbf{k})$ and $(\mathbf{f}, \mathbf{i}$, and I) showing the transverse sections from the distal and proximal of GTs in (d, $\mathbf{g}$, and $\mathbf{j})$, respectively, white lines indicate the planes of sections. $(\mathbf{m}-\mathbf{q})$ showing Shh expression in E14.5 mouse GT. $\mathbf{n}, \mathbf{0}, \mathbf{p}$, and $\mathbf{q}$ are transverse sections of tubercle through the levels labeled with white lines on $\mathbf{~ m}$. Shh expression in E14.5 mouse GT is only retained in urethral epithelium, it stays inside of GT mostly (o, p, and q) except that in the proximal region, which is the future urethral opening site, it extends to the ventral surface of GT. The black arrowheads in $\mathbf{h}$, i, $\mathbf{k}$, I, and $\mathbf{n}$ point to the boundaries of Shh expression domain in ventral epithelium. Stars $\left(^{*}\right)$ in $\mathbf{0}, \mathbf{p}$, and $\mathbf{q}$ indicate the ectoderm covering on the ventral surface of mouse GT. gl glans, hl hindlimb, ta tail, gt, uc, and ue are the same as in Fig.1. Scale bars in a, b, d, g, j, and $\mathbf{m}, 500 \mu \mathrm{m} ; \mathbf{c}, \mathbf{e}, \mathbf{f}, \mathbf{h}, \mathbf{i}, \mathbf{k}$, and I, $200 \mu \mathrm{m} ; \mathbf{n}, 50 \mu \mathrm{m}$, and $\mathbf{0}-\mathbf{q}, 25 \mu \mathrm{m}$.

Sonic hedgehog expression pattern in developing genital tubercle is different between guinea pigs and mice

Shh mRNA is expressed in the endodermal epithelial lining of the cloaca before genital swelling initiation and, later, the urogenital epithelium of the GT. ${ }^{12,19}$ In order to reveal the role of Shh in UG formation, we performed Shh mRNA in situ hybridization on developing guinea pig GTs. The results are shown in Fig. 5. At E22, Shh expression is retained in the whole urethral epithelium, extending from ventral side of GT to the dorsal side (Fig. $5 \mathrm{a}-\mathrm{C}$ ). By E23, differential growth of the dorsal side of the GT has displaced the entire Shh domain to the ventral side of the GT (Fig. 5d). Shh expression domain in guinea pig GT at E23 shows a canal in dorsal and a urethral plate in ventral (Fig. $5 d-f$ ). Consistent with previous observation that the preputial glands in guinea pigs develop late ${ }^{9,}$ we have not detected any Shh expression in the preputial swellings at these early stages. The enlarged glans forms at E25, and Shh expression domain remains in the whole urethra plate and clearly includes the glans portion and distal tip of the urethral plate at E25 and E27 (Fig. 5g-I). In addition, Shh expression domain on the ventral side of E25 and E27 GTs extends out to the ventral surface of GTs (Fig. $5 \mathrm{~g}-\mathrm{I}$ ), and this extension of Shh domain exists from proximal throughout to distal of guinea pig GTs. Interestingly, in E14.5 mouse GT, close to the urethral opening, the similar Shh expression domain extension to the ventral surface of
GTs was also observed, but is only restricted to the urethral opening site in the proximal region (Fig. $5 \mathrm{~m}, \mathrm{n}$ ), not in the middle or distal region of E14.5 mouse GT (Fig. $5 \mathrm{~m}, \mathrm{o}-\mathrm{q}$ ).

\section{DISCUSSION}

As mice and rats do not perform UG formation and distal opening proximal closing processes during external genital development, the literature about how UG forms is very limited. Using guinea pig external genital development model, we discovered that outer layers cell proliferation and inner layers cell death in urethral epithelium, as well as differential cell proliferation between dorsal and ventral regions played important roles during urethral canal movement from dorsal to ventral aspect. After E25, outer layers of urethral epithelial cells at the ventral boundary of the urethral canal proliferate to increase the urethral epithelial cell layers, and then the inner layers of cells perform programmed cell death (apoptosis and autophagy) to enlarge the lumen at the ventral side; thus, the urethral canal subsequently moves to the ventral side (Fig. 6a-d). The enlarged urethral canal moves ventrally gradually, finally it reaches the ventral surface of GT, and then opens to expose the interior of urethral epithelium and form the UG, this last opening step is mainly caused by programmed cell death (Fig. 6e, f). In developing mouse GT, the urethral epithelium 


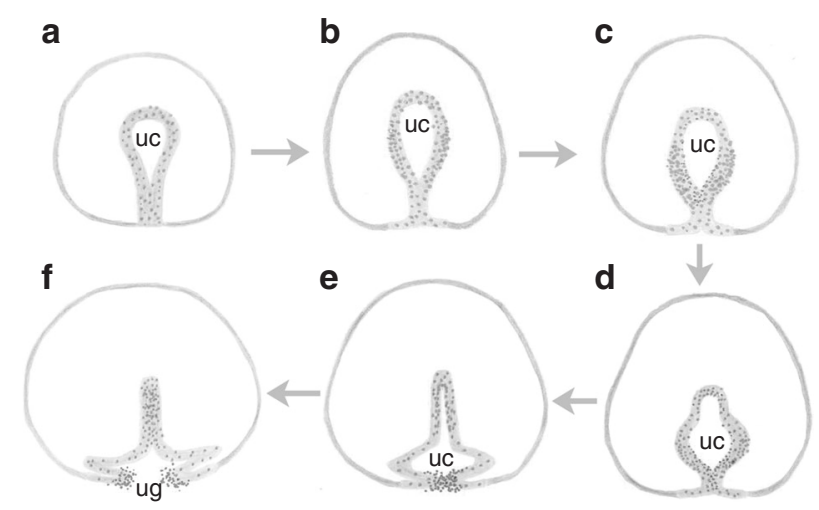

Fig. 6 Developmental basis of UG formation. Schematic drawings of transverse sections of guinea pig GTs with dorsal on the top, showing the urethral canal movement and UG formation. Blue lines in a-f indicate surface epithelium, urethral epithelium, and also Shh mRNA expression domain is in orange, green dots in a-f indicate proliferating cells, red dots in c-f indicate programmed cell death. a-d showing differential cell proliferation and cell death in developing urethral epithelium are important cellular processes in the dorsal-ventral movement of the enlarged urethral canal (uc). Urethral epithelial cells first proliferate to increase the size of the urethral plate and canal (b), then the epithelial cells on the ventral side of UC proliferate to increase epithelial cell layers (c), and programed cell death happens in the inner layers of epithelial cells in the ventral region (c), which causes the enlarged urethral canal to move from dorsal (a) to ventral (d). More cell proliferation in ventral than dorsal, along with the apoptosis in dorsal side may cause the whole urethra to move ventrally (we think the cell proliferation in dorsal region of the mesenchyme also contributes to this process). As this process continues, the canal eventually moves to the ventral margin and programed cell death leads to urethral opening (e), the cell death on both sides of the opening continues, and the whole process repeats from proximal to distal, leading to the distally spreading UG formation (f). The extension of Shh mRNA expression domain to the ventral surface from proximal to distal of GTs may play a key role in UG formation in guinea pigs.

in the distal part forms the urethral plate, but in the proximal region also forms urethral canal-like structure (Fig. 5n-p), suggesting that the initial urethral opening formation may be similar between mice and guinea pigs, the difference is that this opening process in guinea pigs and humans continues from proximal to distal to form the UG, but is restricted to the proximal region to form a proximal urethral opening in mice and rats. Because no UG formation was observed during mouse GT development, different terms were used by researchers to name the process and structures.

Sh mRNA is strongly expressed in endoderm-derived urethral epithelium of multiple species ${ }^{12,21-24}$ and Shh plays important roles in GT outgrowth, regulation of cell survival, and cell cycle. $^{18,19,25,26}$ In mice, Shh expression domain in distal and middle of the GT is retained in urethral epithelium which is restricted to the inside of GT, and only shifts out to the ventral surface of GT at urethral opening site in proximal (Fig. 5n), suggesting that the proximal urethral opening formation may require endoderm-derived Shh-expressing cells to move out to the ventral most region and separate the surface epithelium. The extension of Shh expression domain in urethral epithelium to the ventral surface from proximal throughout to distal in developing GT may play a key role in UG formation in guinea pigs, maybe in humans as well.

In addition, the glans of guinea pig GT can be distinguished at E25, and we have not detected, at any stages studied, an ectodermal ingrowth from the apex of the guinea pig GT. This conflicts with previous reports that the distal urethra forms by apical ectodermal invagination ${ }^{27}$, but is consistent with observations in mouse ${ }^{19}$ and human ${ }^{7,28}$ embryos, as well as the previous fate mapping with $S h h^{\text {Cre }}$ LacZ mice. ${ }^{12}$ Our data indicate that the classical model of urethral development, which assumes that proximal endodermal and distal ectodermal involutions generate the urethra, does not apply to the guinea pigs. We favor a model in which the entire urethra of the guinea pig is derived from endodermal urethral epithelium.

\section{ACKNOWLEDGMENT}

The study was supported by Seed grant from School of Medicine, Southern Illinois University.

\section{ADDITIONAL INFORMATION}

The online version of this article (https://doi.org/10.1038/s41390-018-0236-0) contains supplementary material, which is available to authorized users.

Competing interests: The authors declare no competing interests.

Publisher's note: Springer Nature remains neutral with regard to jurisdictional claims in published maps and institutional affiliations.

\section{REFERENCES}

1. Nelson, C. P. et al. The increasing incidence of congenital penile anomalies in the United States. J. Urol. 174, 1573-1576 (2005).

2. Bouty, A., Ayers, K. L., Pask, A., Heloury, Y. \& Sinclair, A. H. The genetic and environmental factors underlying hypospadias. Sex. Dev. 9, 239-259 (2015).

3. Paulozzi, L. J., Erickson, J. D. \& Jackson, R. J. Hypospadias trends in two US surveillance systems. Pediatrics 100, 831-834 (1997).

4. Nordenvall, A. S., Frisen, L., Nordenstrom, A., Lichtenstein, P. \& Nordenskjold, A. Population based nationwide study of hypospadias in Sweden, 1973 to 2009: incidence and risk factors. J. Urol. 191, 783-789 (2014).

5. Kalfa, N., Philibert, P., Baskin, L. S. \& Sultan, C. Hypospadias: interactions between environment and genetics. Mol. Cell. Endocrinol. 335, 89-95 (2011).

6. Yiee, J. H. \& Baskin, L. S. Environmental factors in genitourinary development. J. Urol. 184, 34-41 (2010).

7. Li, Y. et al. Canalization of the urethral plate precedes fusion of the urethral folds during male penile urethral development: the double zipper hypothesis. J. Urol. 193, 1353-1359 (2015).

8. Overland, M. et al. Canalization of the vestibular plate in the absence of urethral fusion characterizes development of the human clitoris: the single zipper hypothesis. J. Urol. 195, 1275-1283 (2016).

9. Wang, S., Shi, M., Zhu, D., Mathews, R. \& Zheng, Z. External genital development, urethra formation, and hypospadias induction in guinea pig: a double zipper model for human urethral development. Urology 113, 179-186 (2018).

10. Soriano, P. Generalized lacZ expression with the ROSA26 Cre reporter strain. Nat. Genet 21, 70-71 (1999).

11. Harfe, B. D. et al. Evidence for an expansion-based temporal Shh gradient in specifying vertebrate digit identities. Cell 118, 517-528 (2004).

12. Seifert, A. W., Harfe, B. D. \& Cohn, M. J. Cell lineage analysis demonstrates an endodermal origin of the distal urethra and perineum. Dev. Biol. 318, 143-152 (2008).

13. Nieto, M. A., Patel, K. \& Wilkinson, D. G. In situ hybridization analysis of chick embryos in whole mount and tissue sections. Methods Cell Biol. 51, 219-235 (1996).

14. Zheng, Z., Armfield, B. \& Cohn, M. Timing of androgen receptor disruption and estrogen exposure underlies a spectrum of congenital penile anomalies. Proc. Natl Acad. Sci. USA 112, E7194-203 (2015).

15. Fogel, J. L., Thein, T. Z. \& Mariani, F. V. Use of LysoTracker to detect programmed cell death in embryos and differentiating embryonic stem cells. J. Vis. Exp. 68, e4254 10.3791/4254, https://doi.org/10.3791/4254 (2012).

16. Gundersen, H. J. et al. The new stereological tools: disector, fractionator, nucleator and point sampled intercepts and their use in pathological research and diagnosis. APMIS 96, 857-881 (1988).

17. Ormerod, B. K., Lee, T. T. \& Galea, L. A. Estradiol enhances neurogenesis in the dentate gyri of adult male meadow voles by increasing the survival of young granule neurons. Neuroscience 128, 645-654 (2004).

18. Seifert, A. W., Zheng, Z., Ormerod, B. K. \& Cohn, M. J. Sonic hedgehog controls growth of external genitalia by regulating cell cycle kinetics. Nat. Commun. 1, 23 (2010). 
Differential cell proliferation and cell death during the urethral groove... $S$ Wang and Z Zheng

19. Perriton, C. L., Powles, N., Chiang, C., Maconochie, M. K. \& Cohn, M. J. Sonic hedgehog signaling from the urethral epithelium controls external genital development. Dev. Biol. 247, 26-46 (2002).

20. Chikte, S., Panchal, N. \& Warnes, G. Use of LysoTracker dyes: a flow cytometric study of autophagy. Cytom. A 85, 169-178 (2014).

21. Herrera, A. M., Shuster, S. G., Perriton, C. L. \& Cohn, M. J. Developmental basis of phallus reduction during bird evolution. Curr. Biol. 23, 1065-1074 (2013).

22. Larkins, C. E. \& Cohn, M. J. Phallus development in the turtle Trachemys scripta. Sex. Dev. 9, 34-42 (2015).

23. Leal, F. \& Cohn, M. J. Development of hemipenes in the ball python snake Python regius. Sex. Dev. 9, 6-20 (2015).
24. Miyado, M. et al. Expression patterns of Fgf8 and Shh in the developing external genitalia of Suncus murinus. Reproduction 153, 187-195 (2017).

25. Seifert, A. W., Bouldin, C. M., Choi, K. S., Harfe, B. D. \& Cohn, M. J. Multiphasic and tissue-specific roles of sonic hedgehog in cloacal septation and external genitalia development. Development 136, 3949-3957 (2009).

26. Lin, C. et al. Temporal and spatial dissection of Shh signaling in genital tubercle development. Development 136, 3959-3967 (2009).

27. Glenister, T. W. The origin and fate of the urethral plate in man. J. Anat. 88, 413-425 (1954).

28. Hadidi, A. T., Roessler, J. \& Coerdt, W. Development of the human male urethra: a histochemical study on human embryos. J. Pediatr. Surg. 49, 1146-1152 (2014). 